Trauma Berufskrankh 2009 11 [Suppl 2]: 211-216

DOI 10.1007/s10039-008-1464-z

Online publiziert: 7. Februar 2009

(c) Springer Medizin Verlag 2009

\author{
V. Heppert ${ }^{1} \cdot$ P. Herrmann ${ }^{1} \cdot$ P. Thoele ${ }^{1} \cdot$ M. Pelzer ${ }^{2} \cdot$ C. Wagner ${ }^{3}$ \\ ${ }^{1}$ Abteilung Septische Chirurgie Knochen-, Gelenk- und Protheseninfektionen, \\ BG-Unfallklinik Ludwigshafen \\ ${ }^{2}$ Klinik für Hand-, Plastische und Rekonstruktive Chirurgie - \\ Schwerbrandverletztenzentrum, BG-Unfallklinik Ludwigshafen \\ ${ }^{3}$ Klinik für Unfallchirurgie und Orthopädie, BG-Unfallklinik Ludwigshafen
}

\title{
Weichteilmanagement und knöcherne Rekonstruktion
}

Anzahl der VVS die DRG-Vergütung exponentiell zunimmt.

Von einer anderen Warte aus betrach-

Das Problem, mit dem wir uns im vorliegenden Beitrag beschäftigen, zählt zu den schwierigsten im Gebiet der rekonstruktiven Chirurgie. Grundsätzlich müssen mehrere Fachgebiete beteiligt sein, der Zugang zum Problem muss immer multidisziplinär angelegt sein [13, 15, 16]. Dies macht aber nur dann Sinn, wenn bereits zu Beginn der Behandlung ein entsprechendes Gesamtkonzept erstellt wird [8].

Die Vielfalt der Komplikationsmöglichkeiten während des Krankheitsverlaufs bei Frakturen mit Weichteilschaden erfordert eine ständige Überprüfung und ggf. Korrektur des eingeschlagenen Weges. Auch die therapeutische Korrektur ist wiederum von den einzelnen Fachgebieten individuell zu überprüfen. Eine falsche Strategie ist in derartigen Fällen immer besser als überhaupt keine Strategie, denn falsch angedachte Lösungen zeigen ihre Probleme bei sorgfältiger klinischer Kontrolle rasch und können dann korrigiert werden. Abwarten und nichts tun ist für den Patienten definitiv mit keinem Vorteil verbunden und demzufolge kontraproduktiv. Leider sind dennoch endlose Vakuumversiegelungen (VVS) ohne jedes weiterführende Konzept klinischer Alltag. Nach einer Odyssee des Patienten wird dieser meist erst am Schluss in ein septisches Zentrum überwiesen. Dies kostet viel Geld und nützt niemandem außer der behandelnden Klinik, da paradoxerweise abhängig von der tet kann eine kurz angedachte Amputation einen Patienten in die persönliche Katastrophe führen, seelisch, beruflich und privat. In diesem Kontext müssen folgende Fragen erlaubt sein:

- Kann und darf wirklich jeder behandelnde Arzt die Entscheidung zu einer Amputation treffen?

- Ist es nicht besser, bei einer solch schwer wiegenden Entscheidung eine 2. Meinung einzuholen?

- Sollte in solchen Komplexfällen - vitale Indikation natürlich ausgeschlossen - nicht nur derjenige die Indikation zur Amputation stellen, der die heutigen Möglichkeiten des Extremitätenerhaltes kennt?

\section{Weichteile}

„Osteomyelitis is infected dead bone, surrendered by an envelope of compromised soft tissue"

so die Aussage von George Cierny III [2, 3]. Besser kann man das Problem nicht beschreiben. Der Infekt des Knochens ist nahezu immer mit schwierigen Weichteilverhältnissen vergesellschaftet (• Abb. 1).

Sie resultieren aus:

- dem Trauma

- den Voroperationen

- der chronischen Infektsituation

- regionalen bzw. systemischen Durchblutungsstörungen.

\section{Trauma}

Es steht immer am Anfang der Behandlungskette. Lokale Kontusionen der Haut führen zu instabilen Narben und regionalen Abflussstörungen, die häufig an einer Braunverfärbung der Haut durch Pigmenteinlagerungen $\mathrm{zu}$ erkennen sind (• Abb. 1b). Diese Weichteile neigen bei Revisionseingriffen zu Wundkomplikationen. Bei der Indikation zu Folgeeingriffen muss dies unbedingt bedacht werden.

\section{Voroperationen}

Jede Operation hinterlässt Narbengewebe, welches insbesondere nach multiplen Voreingriffen den Zugang zum Knochen ungemein erschwert. Hierdurch steigt das Risiko von Wundheilungsstörungen. Erforderliche notwendige Eingriffe am Knochen (z. B. Osteosynthesen, Spongiosaplastiken) werden daher zu vermeiden versucht, der Heilungsverlauf verlängert sich [17].

\section{Chronische Infektsituation}

Beim akuten Infekt ist die gerötete Haut sehr vulnerabel. Eine einheitliche Klassifikation existiert noch nicht [16].

Beim chronischen Infekt finden sich manchmal $1 \mathrm{~cm}$ dicke Narbenplatten über dem Knochen. In diesem Narbengewebe sind in der pathologischen Untersuchung alle Zellen zu finden, die die Infektion unterhalten. Ohne Sanierung dieser Weichteile wird eine Infektberuhigung nur schwer möglich sein. 


\section{Behandlungsalgorithmen}
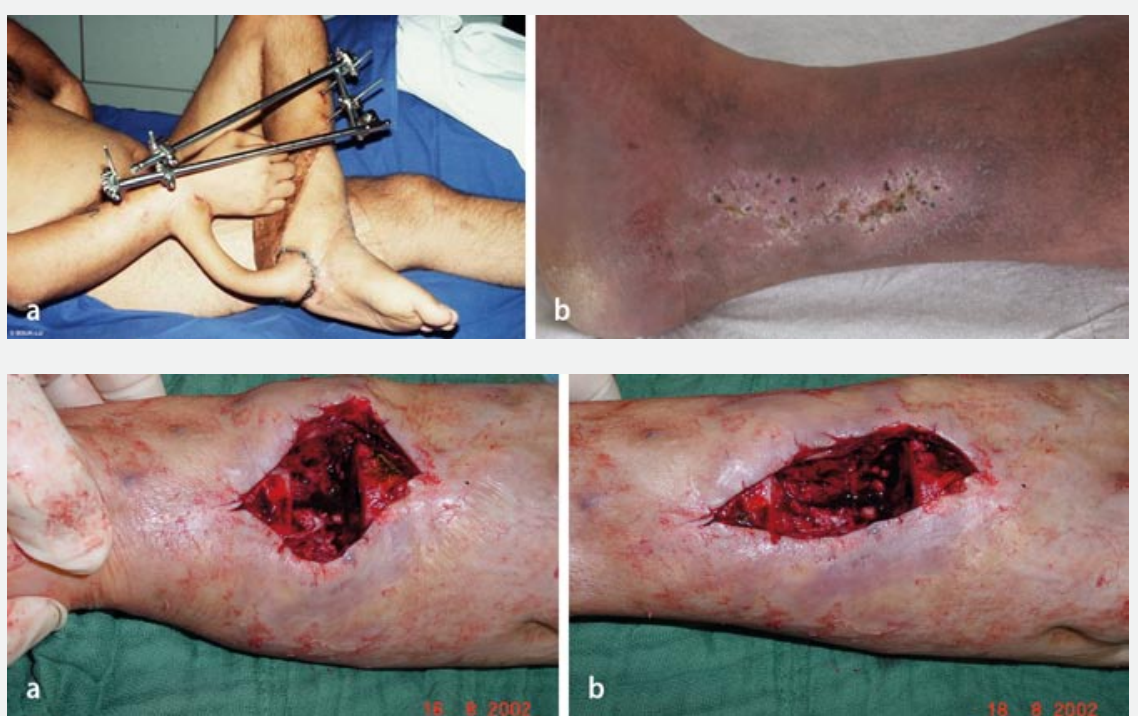

Abb. 14 a Notwendigkeit einer erneuten Lappenplastik nach Nekrose der Spitze am M.-latissimus-dorsi-Lappen, Lösung des Problems durch Rollstiellappen (heutzutage nur noch sehr seltene Ausnahmeindikationsstellungen), b schwere Störung der Hauttrophik bei chronischer Osteitis

Abb. 24 a Defektsituation von Knochen und Weichteilen, $\mathbf{b}$ bei longitudinaler Wunde: nicht verschließbare Wunde als Folge der Extremitätenverkürzung
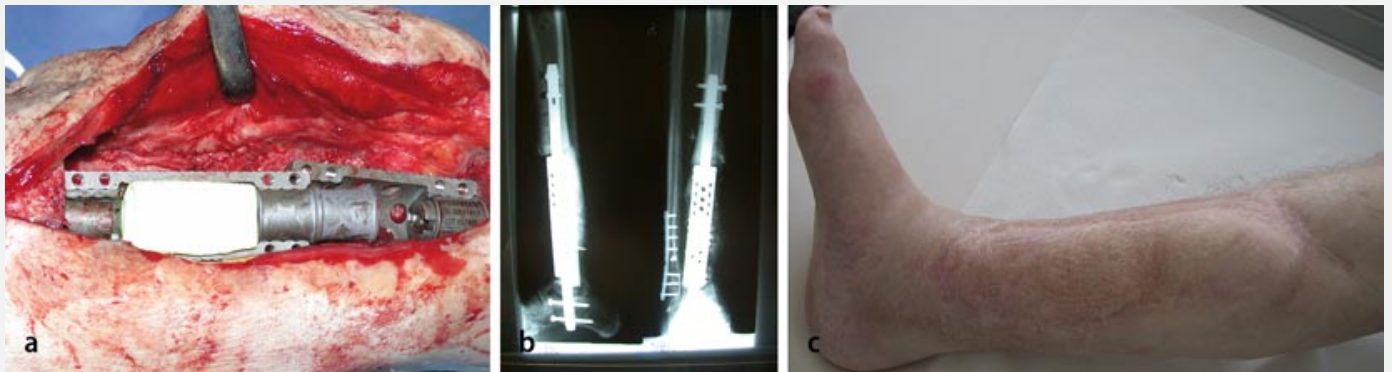

Abb. $3<$ a Wiederherstellung der Stabilität durch Osteobridge ${ }^{\circledast}$ bei 68 -jährigem Patienten nach erfolglosem Versuch mit freier Fibula, b radiologisches, c klinisches Ausheilungsergebnis
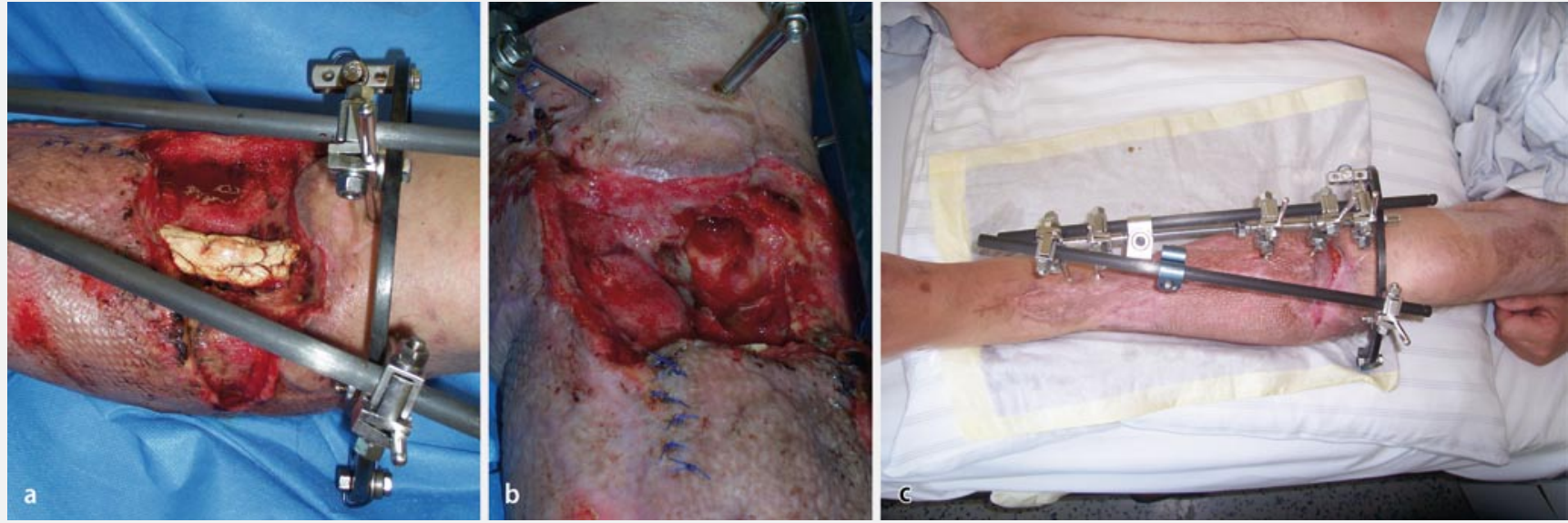

Abb. $4 \Delta$ a Chronische Osteitis der Tibia, Zustand nach 4 gescheiterten Lappenplastiken, proximaler Unterschenkel mit einliegendem Spacer, b Defektsitus nach Débridement, c verschlossene Wunde nach offenem Segmenttransport von distal in den Tibiakopf, 4 Monate später Fixateurabbau ohne weitere Folgeeingriffe

\section{Regionale bzw. systemische Durchblutungsstörungen}

Sie fördern ebenfalls die Wundheilungsstörung. Eine Gefäßdiagnostik (Pulse, Doppler-, bildgebende Verfahren) sollte bei zweifelhaften Befunden immer durchgeführt werden. Gegebenenfalls sind vor der Lappenplastik gefäßchirurgische Eingriffe indiziert.

\section{Knochen}

Grundsätzlich sollte jeder infizierte Knochenanteil entfernt werden, um - ähnlich der Tumorchirurgie - größtmögliche Sicherheit zu erzielen [12]. Dieser Radikalität sind aber durch individuelle Befunde und das Risikoprofil des Patienten Grenzen gesetzt: Kürzere Defekte kann man mit einer Extremitätenverkürzung sanie- ren [16], Defekte von 3-4 cm werden nahezu überall mit Spongiosaplastik überbrückt, größere Defekte mit dem IlisarovVerfahren [16, 19], der freien Fibula oder so genannten Compound Flaps [7].

Extremitätenverkürzung. Bei einem Knochendefekt von bis zu maximal $3 \mathrm{~cm}$ ist die Verkürzung einer Extremität mit dem Patienten zu diskutieren. Dabei han- 
delt es sich zweifelsfrei um das Verfahren mit der kürzesten Heilungszeit. Es darf aber in Kombination mit einem Weichteildefekt nicht vergessen werden, dass longitudinale Defekte durch diese Maßnahme breiter werden und sich ggf. die Wunde dann nicht verschließen lässt (• Abb. 2). Horizontale Wunden lassen sich auf diese Art und Weise sehr gut sanieren [14]. Man sollte allerdings nie wertvollen Knochen kürzen, nur um eine Lappenplastik zu vermeiden.

Autogene Spongiosaplastik. Sie stellt nach wie vor den „Goldstandard“ für Defekte bis $4 \mathrm{~cm}$ dar $[13,19]$. Die Heilungszeit ist hierbei kürzer, als mit einem Transportverfahren anzusetzen wäre. Limitierend ist die Frage, wie viel Spongiosa nach multiplen gescheiterten Voroperationen noch vorhanden ist. Da es auch dem erfahrendsten septischen Chirurg nur gelingen kann, ein infiziertes Gebiet in ein kontaminiertes zu überführen, mischen wir die Spongiosa grundsätzlich mit einem antibiotikabeladenen Kollagenschwamm. Bei Schaftdefekten ist es sinnvoll, einen solchen Schwamm aufzurollen und proximal und distal in die Markhöhle einzuführen. Bei Platzierung der Spongiosa um diesen Schwamm ergeben sich 3 Vorteile:

1. Es muss weniger Spongiosa entnommen werden.

2. Man erhält einen Röhrenknochen mit offener Markhöhle.

3. Der reduzierte Durchmesser der Plastik „garantiert" einen besseren Anschluss an die einwachsenden Gefäße. Bei sehr ausgedehnten Spongiosaplastiken kann es zur Sequesterbildung von Spongiosaanteilen im Zentrum der Plastik kommen, da hier der Gefäßanschluss nicht oder zu spät stattfindet.

Die offene Spongiosaplastik ist im Zeitalter differenzierter Lappenplastiken kein probates Verfahren mehr $[16,18]$. Bei ausgedehnteren Spongiosaplastiken und wenn man infolge leerer Spenderstellen auf Bankspongiosa angewiesen ist, hat sich bei uns das Gravitational Platelet System $\left(\right.$ GPS $^{\text {rm }}$-System ${ }^{\circledR}$ ) bewährt. Die im Blut des Patienten zirkulierenden Thrombozyten sind Träger von Wachstumsfaktoren, die den Heilungsprozess initiieren sollen. Mit

Trauma Berufskrankh 2009 · 11 [Suppl 2]: 211-216 DOI 10.1007/s10039-008-1464-z

(C) Springer Medizin Verlag 2009

\section{Heppert · P. Herrmann · P. Thoele - M. Pelzer · C. Wagner Weichteilmanagement und knöcherne Rekonstruktion}

\section{Zusammenfassung}

Auf dem Gebiet der Frakturbehandlung wurden in den letzten Jahrzehnten erhebliche Fortschritte erzielt. Gleiches gilt für die Entwicklung plastisch-rekonstruktiver Verfahren zum Verschluss von Weichteildefekten. Aber die sinnvolle Kombination dieser eigentlich perfekt aufeinander abgestimmten Verfahren im klinischen Alltag lässt leider ideale Gesamtkonzepte zur Ausnahme werden. Kontraproduktive Abrechnungsverfahren wie die "diagnosis related groups" (DRG) werten eine Amputation häufig günstiger für eine Klinik als den Erhaltungsversuch einer geschädigten Extremität. Aber auch Informationslücken der Ärzte/-innen über die heute gängigen Verfahren der anderen Gebiete sind nicht wegzudiskutieren. Die Spezialisierung wird häufig so weit vorangetrieben, dass ein Gesamtüberblick und daraus resultierend ein Gesamtkonzept fehlen. Die Ursachen dafür sind mannigfaltig, aber nicht mit persönlichem Desinteresse zu erklären. Überlastung und stupide EDV-Tätigkeiten sind hier führend zu nennen, da diese den Arbeitsalltag zunehmend weg vom Patienten orientieren. Ziel dieser Arbeit ist es, auf möglichst einfach nachvollziehbarem Weg Grundkenntnisse in der Lösung schwieriger Komplexfälle zu vermitteln.

\section{Schlüsselwörter}

Osteitis · Weichteilschaden · Erhaltungsversuch · Lappenplastik · Komplikation

\section{Soft tissue management and osseous reconstruction}

\section{Abstract}

Fracture treatment has seen much progress during the last decades, as has plastic reconstructive surgery. New flaps were found for closing soft tissue defects, and we can now say that there is nearly no defect that cannot be closed. But the combination of these perfectly fitting medical solutions is not perfect at all. Every patient with a complex situation involving infection needs an individualized plan of care right from the beginning, and this should always be developed via a multidisciplinary approach. However, this concept is still an exception in our country. The DRG system is counterproductive; for instance, in most cases the payment for amputation is better than for reconstruction. In addition, there is still a lack of knowledge among doctors making referrals to those in other disciplines, and the gap is increasing; more and more specialization is sometimes a poor concept because at the end, there is nobody who is familiar with the whole problem. Moreover, difficult working conditions, computer work, and documentation take physicians further and further away from the patient. The goal of this paper is to raise interest in this situation and to offer solutions to my colleagues in a very simple way.

\section{Keywords}

Osteomyelitis · Soft tissue defect · Reconstruction · Flap transfer · Complication 


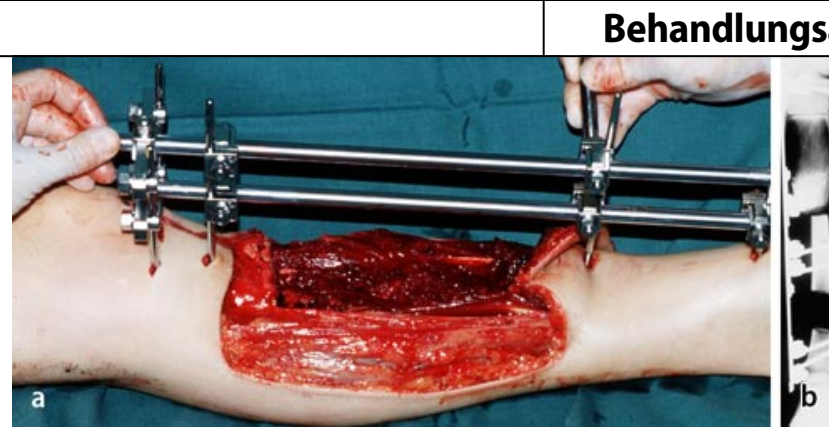

\section{algorithmen}
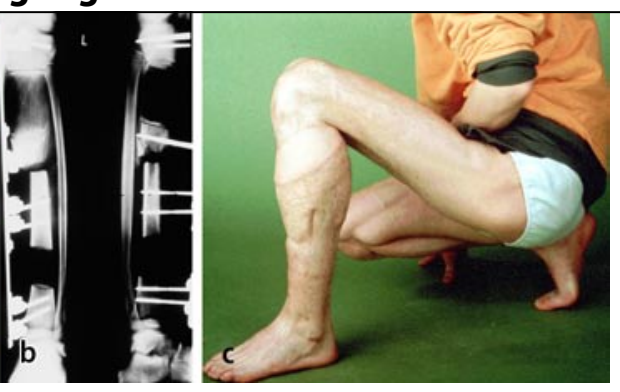

Abb. $5 \triangleleft$ a $16 \mathrm{~cm}$ langer Knochen- und Weichteildefekt am Unterschenkel, b bisegmentaler Transport nach M.-latissimus-dorsiLappen, c Ausheilungsbild 16 Monate später
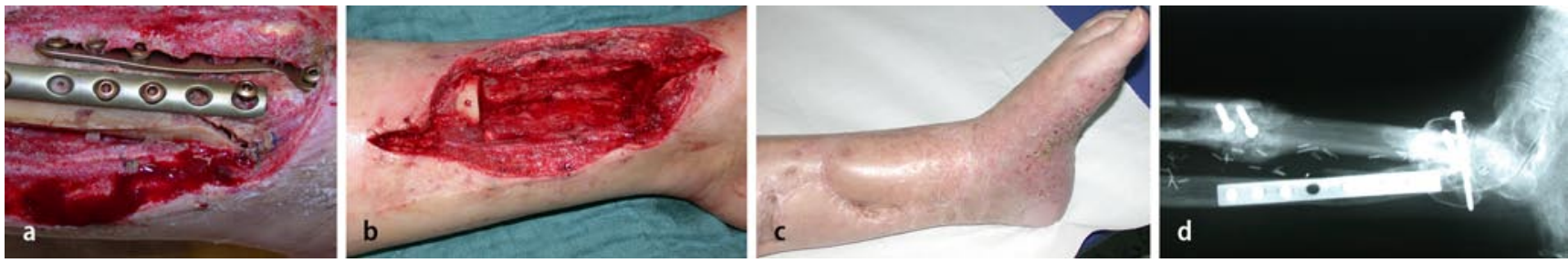

Abb. $6 \Delta$ a Nekrose der Tibia nach distaler Unterschenkelfraktur einer 72-jährigen Frau, b Situs nach radikalem Débridement, c klinisches, $\mathbf{d}$ radiologisches Ausheilungsbild

Hilfe des GPS $^{\text {rw }}$-Systems ${ }^{\circledast}$ werden diese Wachstumsfaktoren aus dem Patientenblut - der Patient erhält somit kein Fremdmaterial - hochkonzentriert gewonnen. Das Verfahren geht mit deutlich verkürzten knöchernen Heilverläufen einher.

Ilisarov-Verfahren. Natürlich kann man mit dem Verfahren nach Ilisarov [20] grundsätzlich nahezu jeden Defekt überbrücken. Die Entscheidung für diese Methode ist aber nicht nur eine Frage der Behandlungsdauer:

- Zunehmende Pininfektionen stellen ein Risiko dar, welches primär während des Transports als hoch einzuschätzen ist. Je länger jedoch die Ausreifung dauert, desto eher kommt es zu Pinlockerungen und zunehmenden Infektionen [10, 20].

- Abhängig von der Defektgröße und Lokalisation lässt sich in manchen Fällen kein Transportsegment mehr bilden, welches eine ausreichende Durchblutung garantiert. Bei sehr langen Schaftdefekten kann dies ebenso vorkommen wie nach gescheitertem Transport infolge von Regeneratversagen.

- Ab einem Alter von etwa 65 Jahren ist das Verfahren nicht mehr als sicher anzusehen, die übliche Rate von $6 \%$ Regeneratversagen $[11,19]$ steigt dann exponentiell an. Alternative Verfahren sind anzudenken (• Abb. 3).

- Da beim Ilisarov-Verfahren Knochen und Weichteile transportiert werden, lassen sich Weichteildefekte allein durch den Segmenttransport sicher verschließen [17, 21]. Hierbei ist aber darauf zu achten, dass die Knochenendigungen während des Transports von Weichgewebe bedeckt sein müs$\operatorname{sen}(\bullet$ Abb. 4, [21]).

\section{Lappenplastiken}

In den letzten Jahren wurden hochdifferenzierte neue Techniken entwickelt $[5,6]$. Für die Infektberuhigung der Osteitis ist es völlig unerheblich, ob man einen fasziokutanen oder einen Muskellappen wählt [9]. Dennoch muss diese Wahl differenziert betrachtet werden. Insbesondere bei größeren Höhlenbildungen bieten die Muskellappen durch ihr größeres Volumen und die perfekte Durchblutung Vorteile. Die fasziokutanen Lappen sind schneller autonom, sodass Folgeoperationen am Knochen leichter durchführbar sind [9]. So kann z. B. ein M.-latissimus-dorsi-Lappen in den ersten Monaten nach dem Transfer so ödematös anschwellen, dass eine Mobilisierung für einen Folgeeingriff am Knochen nicht möglich ist. Ist z. B. eine Plattenosteosynthese geplant, lässt sich häufig trotz ,eingeschobener Technik“ der Defekt über der Platte nicht verschließen.

Aus diesem Grund richtet sich die Indikation nach:

- Größe und Form des Defekts

- Ort des Defekts

- am Knochen geplanten Folgeoperationen - Diese müssen vor der Lap- penplastik geklärt werden, da der ausschließliche Defektverschluss bei den heutigen Möglichkeiten nicht mehr ausreichend ist.

- Vermeidung instabiler Narben für die Zukunft insbesondere in Zonen mechanischer Belastung - Deshalb ist mit der Indikation zur Spalthaut in entsprechenden Arealen eher $\mathrm{Zu}$ rückhaltung geboten.

- Risikoprofil des Patienten - Dabei kommt dem Nikotin wesentlich höhere Bedeutung als dem Alter bzw. dem Diabetes mellitus zu.

- Morbidität des Hebedefekts - Sie muss in die Indikation stets mit einfließen. Der Ausfall des N. suralis z. B. beim Suralislappen ist grundsätzlich Folge dieser Plastik. Auch proximale Stumpfneurome dürfen in ihrem Schaden für den Patienten nicht außer Acht gelassen werden. So hinterlässt jeder Lappen seinen typischen Folgeschaden.

- Kosmetik - Sowohl im Hebe- als auch im Empfängerbereich stellt diese Frage höchste Ansprüche an die Operateure. Eingehen in diese Indikation muss auch das Berufsprofil der Patienten.

\section{Vorgehen}

Bei der Therapie der oben angeführten Folgezustände hat sich ein phasengerechtes Vorgehen bewährt [13, 16, 19]: 
Phase des Débridements. Alles infizierte Gewebe mit Ausnahme vitaler Strukturen wie Nerven und Gefäßen wird entfernt (- Abb. 5a). Nekrotische Sehnen werden ebenfalls reseziert. Der Situs wird in unserer Klinik nach Débridement mit der Jet-Lavage gereinigt, danach wird in der Regel ein lokaler Antibiotikumträger eingelegt. Eine temoporäre VVS wird zur Optimierung des Wundgrundes angelegt. Der Knochendefekt wird zumeist über Fixateur stabilisiert.

Phase der Weichteilsanierung. Nach nochmaligem Débridement wird die Wunde durch Lappenplastik saniert. Ein lokaler Antibiotikumträger wird wieder eingelegt.

Phase des Knochenaufbaus. Der eingeheilte Lappen wird an der sichersten Stelle (Cave Lappengefäß) gehoben, nach Entfernung des Lokalantibiotikumträgers wird eine Spongiosaplastik wie oben ausgeführt vorgenommen. Der Zeitpunkt richtet sich nach den klinischen und laborchemischen Entzündungswerten.

Bei einer Kallusdistraktion werden nach Lappeneinheilung der Fixateur umgebaut und die Kortikotomie vorgenommen ( $\bullet$ Abb. 5b,c).

\section{Ausnahmen}

Offener Segmenttransport. Plant man einen solchen, bleibt die débridierte Wunde offen, ein Lokalantibiotikum findet dann keine Anwendung, die Kortikotomie erfolgt in der gleichen Operation. Phase 2 und Phase 3 entfallen. Die Wunde wird täglich geduscht, ab Tag 7 wird mit dem Transport begonnen.

Notwendigkeit einer internen Osteosynthese. Zeigt es sich, dass eine interne Osteosynthese unverzichtbar ist, kann es möglich sein, dass Phase 1 und 2 einzeitig stattfinden, damit dass Implantat sofort von gut durchblutetem Lappen bedeckt ist. Unsere Erfahrung belegt, dass ein einmal infiziertes Implantat nie mehr steril wird $[1,4]$. Es ist in solchen Fällen besser, das Implantat nach gründlichem Débridement durch ein neues Implantat zu ersetzen. Ob dann die VVS eingesetzt oder sofort die Lappenplastik kombiniert mit Lo-

Abb. 7 2 Jahre nach

M.-latissimus-dorsiLappen, weiterhin sequestrierte Tibia nach mehreren frustranen Osteosynthesen, keine Verbindung zwischen Lappen und Knochen eingetreten

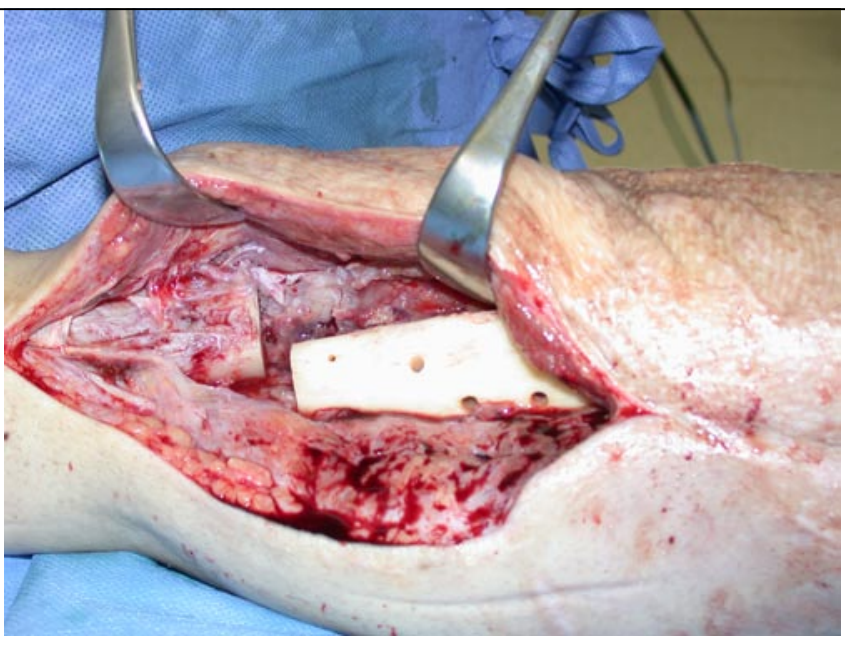

kalantibiose durchgeführt werden, ist Geschmackssache.

\section{Weitere Ausnahmen.}

- Bei osteokutanen Lappen, bei denen Knochen- und Weichgewebedefekte zusammen saniert werden, ist diese Phasentherapie nicht aufrecht zu erhalten. Hier sind Einzelfallentscheidungen gefragt (• Abb. 6).

- Bei Infektpersistenz muss Phase 1 wiederholt werden.

\section{Diskussion}

Die Behandlung von infizierten kombinierten Weichgewebe- und Knochendefekten ist sehr komplex und sollte nur in darauf spezialisierten Zentren erfolgen $[12,16]$. Erst wenn das gesamte rekonstruktive Spektrum vorgehalten werden kann, sind individuell optimierte Ergebnisse für jeden Patienten und verkürzte Krankheitsverkäufe für die Kostenträger zu erzielen. Dem Débridement kommt nach wie vor die Schlüsselstellung zu. Es bedeutet für einen Patienten nur Zeitverzögerung, wenn auf einen denudierten Knochen eine Lappenplastik gesetzt wird, da es in diesem Fall in jedem Fall zu Pseudarthrosen bzw. Infektrezidiven kommt (• Abb. 7).

Eine 24-stündige mikrochirurgisch erfahrene Mannschaft muss zur Verfügung stehen, da eine Anastomosenthrombose einen Notfall darstellt, der sofort operativ angegangen werden muss. Nur die ganz frühe Zusammenarbeit der verschiedenen Fachgebiete garantiert bestmögliche Ergebnisse.

\section{Korrespondenzadresse \\ Dr. V. Heppert \\ Abteilung Septische Chirurgie Knochen-, Gelenk- und Protheseninfektionen, BG-Unfallklinik Ludwigshafen Ludwig-Guttmann-Straße 13 67071 Ludwigshafen \\ Vocalila.hep@t-online.de}

Interessenkonflikt. Der korrespondierende Autor gibt an, dass kein Interessenkonflikt besteht.

\section{Literatur}

1. Arens S, Hansis M, Schlegel U et al (1996) Infection after open reduction and internal fixation with dynamic compression plates - Clinical and experimental data. Injury [Suppl 3] 27:27-33

2. Cierny G III (1990) The classification and treatment of adult osteomyelitis. In: Evarts CM (ed) Surgery of musculoskeletal systems, 2nd edn. Churchill Livingstone, New York

3. Cierny G III, Mader JT (1984) Adult chronic osteomyelitis. Orthopaedics 7:1557

4. Cordero J, Munuera L, Folgueira M (1996) The influence of the chemical composition and surface of the implant on infection. Injury [Suppl 3] 27:3437

5. Dragu A, Jeffries J, Bach A, Horch R (2008) Prinzipien der Lappenplastiken: Eine Übersicht. CHAZ, 9. Jahrg. 2. Heft, 59-66

6. Hallock G (2003) Direct and indirect perforator flaps: the history and the controversy. Plast Reconstr Surg 111:855-865

7. Hallock GG (2006) Further clarification of the nomenclature for compound flaps. Plast Reconstr Surg 117:151-160

8. Heppert V, Wentzensen A (1995) Der Stellenwert der Weichteilsanierung im Behandlungskonzept der Osteitis. In: Jerosch J (Hrsg) Infektionen des Bewegungsapparates. Thieme, Stuttgart, S 74-78

9. Heppert V, Wentzensen A (1995) Myocutaneous versus fasciocutaneous flaps in the treatment of lower leg osteitis. Eur J Ortop Surg Traumatol 5(1):27-31

10. Heppert V, Wentzensen A (1998) Der „Pin-track-Infekt" nach Fixateur externe Osteosynthese. OP J 14 136-145 
11. Heppert $\mathrm{V}$, Wentzensen $A$ (2000) Kallusdistraktion am Unterschenkel - Erfahrungen einer Lernkurve. Osteosynthese Int [Suppl 1] 8:5-7

12. Heppert V, Wentzensen A (2002) Osteitis Unterschenkel. Trauma Berufskrankh 4:114-120

13. Heppert V, Wentzensen A (2002) Prinzipien der operativ-chirurgischen Therapie der Osteitis. Trauma Berufskrankh 4:321-328

14. Heppert V, Wentzensen A (2006) Weichteildefekte - Grundlagen. In: Ewerbeck V, Wentzensen A, Holz $\mathrm{F}$ et al (Hrsg) Standardverfahren in der operativen Orthopädie und Unfallchirurgie, 2. Aufl. Thieme, Stuttgart

15. Heppert V, Wagner C, Glatzel U, Wentzensen A (2002) Prinzipien der operativ-chirurgischen Therapie der Osteitis. Trauma Berufskrankh 4:321-328

16. Hofmann G (2004) Chronische Osteitis. Infektionen der Knochen und Gelenke. Urban \& Fischer, München, S 61-83

17. Josten C, Muhr G (1999) Der Tibiasegmenttransport. Unfallchirurg 102:514-524

18. Papineau L, Alfageme A, Dalcourt JP, Pilon L (1979) Osteomyelite chronique: excision et greffe de spongieux a l'air libre apres mises a plat extensives. Int Orthop 3:165-178

19. Schmidt $H$, Hadler $D$, Wurm $M$, Jürgens $C$ (2003) Therapie der Infekt-/Defekt-Pseudarthrosen der Unteren Extremitäten. Trauma Berufskrankh [Suppl 2] 5:318-327

20. Schmidt H, Kranz HW, Wittek F, Jürgens C (1995) Interne und externe Stabilisierung bei der Therapie der Osteomyelitis? In: Jerosch J (Hrsg) Infektionen des Bewegungsapparates. Thieme, Stuttgart, S68-74

21. Suger G, Fleischmann W, Hartwig E, Kinzl L (1995) Der offene Segmenttransport. Unfallchirurg 98:381-385 